\title{
Sternohyoid muscles plication and sternocleidomastoid muscles rejuvenation in neck lift: a retrospective study of 1,019 consecutive patients
}

\author{
Luiz A. Auersvald, André Auersvald \\ Department of Plastic Surgery, Clínica Auersvald de Cirurgia Plástica, Alameda Presidente Taunay, Curitiba 1756, Paraná \\ 80430-000, Brazil. \\ Correspondence to: Luiz A. Auersvald, Department of Plastic Surgery, Clínica Auersvald de Cirurgia Plástica, Alameda Presidente \\ Taunay, Curitiba 1756, Paraná 80430-000,Brazil. E-mail: luizauersvald@uol.com.br \\ How to cite this article: Auersvald LA, Auersvald A. Sternohyoid muscles plication and sternocleidomastoid muscles rejuvenation \\ in neck lift: a retrospective study of 1,019 consecutive patients. Plast Aesthet Res 2021;8:4. \\ http://dx.doi.org/10.20517/2347-9264.2020.187
}

Received: 8 Oct 2020 First Decision: 23 Nov 2020 Revised: 30 Nov 2020 Accepted: 14 Dec 2020 Published: 8 Jan 2021

Academic Editor: Raúl González-García Copy Editor: Miao Zhang Production Editor: Jing Yu

\begin{abstract}
Aim: Improving the cervical contour is one of the main goals of patients seeking face and neck rejuvenation. However, little attention has been dedicated to refine the anterior border of the sternocleidomastoid muscles and to improve the inferior neck. In this study, the authors sought to describe new surgical tactics to address these issues.
\end{abstract}

Methods: The records of 1,019 patients were evaluated retrospectively. Surgical strategies to treat the neck were reviewed and two new approaches, described. Plication of the sternohyoid muscles and lateral plication of the platysma along the anterior border of the sternocleidomastoid muscles are detailed and supplemented with a video.

Results: Of the 1,019 patients, 937 patients (91.9\%) underwent subplatysmal neck lift. Three-hundred-and-fortyeight patients (34.1\%) underwent sternohyoid muscles plication, and 784 patients (76.9\%) had rejuvenation of the sternocleidomastoid muscles. The most common complication was weakness of the lower lip depressors $[79$ patients $(7.7 \%)$.

Conclusion: Approaching the sternohyoid muscles and rejuvenating the sternocleidomastoid muscles are new reliable and effective tactics to optimize results in neck lifts.

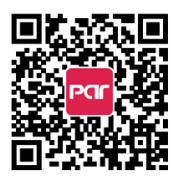


Keywords: Neck lift, face lift, sternohyoid muscle, sternocleidomastoid muscle, quilting sutures, hematoma, platysmaplasty, platysma

\section{INTRODUCTION}

Improving the cervical contour is one of the main goals of patients seeking face and neck rejuvenation ${ }^{[1-4]}$. Criteria to define the youthful neck have been previously established and include a distinct inferior mandibular border, an identifiable subhyoid depression, a visible thyroid cartilage bulge, a cervicomental angle between $105^{\circ}$ and $120^{\circ}$, and a discernable anterior border of the sternocleidomastoid muscles (SCMs) ${ }^{[5]}$. The musculomandibular triangle, located between the superior aspect of the SCM and the posterior margin of the ramus of the mandible, is another important mark of the young neck ${ }^{[6]}$.

Subplatysmal and supraplatysmal fat deposits, hypertrophied anterior bellies of the digastric muscles (DMs), enlarged and ptotic submandibular salivary glands (SMGs), and skin laxity progressively reduce the clear presence of the aforementioned parameters.

Poor definition of the inferior neck (i.e., the cervical area below the hyoid bone) is also a frequent complaint of patients and reflects redundance of skin, accumulation of subcutaneous fat, and infrahyoid muscles flaccidity, especially that of the sternohyoid muscles (SHMs). In fact, patients have become more aware of these alterations because of the use of mobile phones for video calls. Flexing the neck during these calls intensifies the self-perception of cervical aging [Figure 1] ${ }^{[7]}$.

Surgical techniques to rejuvenate the neck have been described in detail ${ }^{[6-13]}$. However, little attention has been dedicated to approaches that aim at improving the inferior neck and rescuing a lost SCM anterior border. Herein, we describe two new tactics dedicated to solving these issues.

\section{METHODS}

A retrospective cohort study of the records of 1,019 consecutive patients who underwent neck lift, performed by the same team of surgeons and anesthesiologists between September 2011 and July 2020, was conducted. Quantitative results were collected and analyzed. Considering that the main goal of this work is to present a detailed description of the subplatysmal technique, including two novel tactics (the SHMs plication and the SCMs rejuvenation), subjective data were not retrieved. Informed consent about the surgical procedure and its risks and benefits was obtained from all patients. This study was conducted in accordance with the principles of the Declaration of Helsinki.

\section{Anesthesia}

Patients undergo total intravenous anesthesia with orotracheal intubation. They are initially induced with midazolam $(0.1 \mathrm{mg} / \mathrm{kg})$, methadone $(0.1 \mathrm{mg} / \mathrm{kg})$, propofol (target-controlled infusion - $2.5 \mathrm{mg} / \mathrm{mL}$ ), and rocuronium bromide ${ }^{[4]}$, concomitantly followed by either remifentanil (target-controlled infusion $-5 \mathrm{ng} / \mathrm{mL}$ ) or dexmedetomidine ( $1 \mathrm{mg} / \mathrm{kg}$ for $10 \mathrm{~min}$ ). A local anesthetic tumescent infiltration is used to facilitate dissection and to improve analgesic control during and after surgery [ $1 \mathrm{~L}$ of saline, $40 \mathrm{~mL}$ of $2 \%$ lidocaine, $20 \mathrm{~mL}$ of $1 \%$ ropivacaine, and epinephrine $(1: 1,000,000)]$.

Patients are then kept on propofol (target-controlled infusion) and either remifentanil (lowest possible dose after local anesthesia) or dexmedetomidine (0.2 to $0.7 \mathrm{ng} / \mathrm{kg} / \mathrm{h}$ ).

Nausea and vomiting are prevented by using dexamethasone $(8 \mathrm{mg})$ and ondansetron $(8 \mathrm{mg})$, both in intravenous infusion during anesthesia induction and $4 \mathrm{mg}$ of intravenous ondansetron is administered 

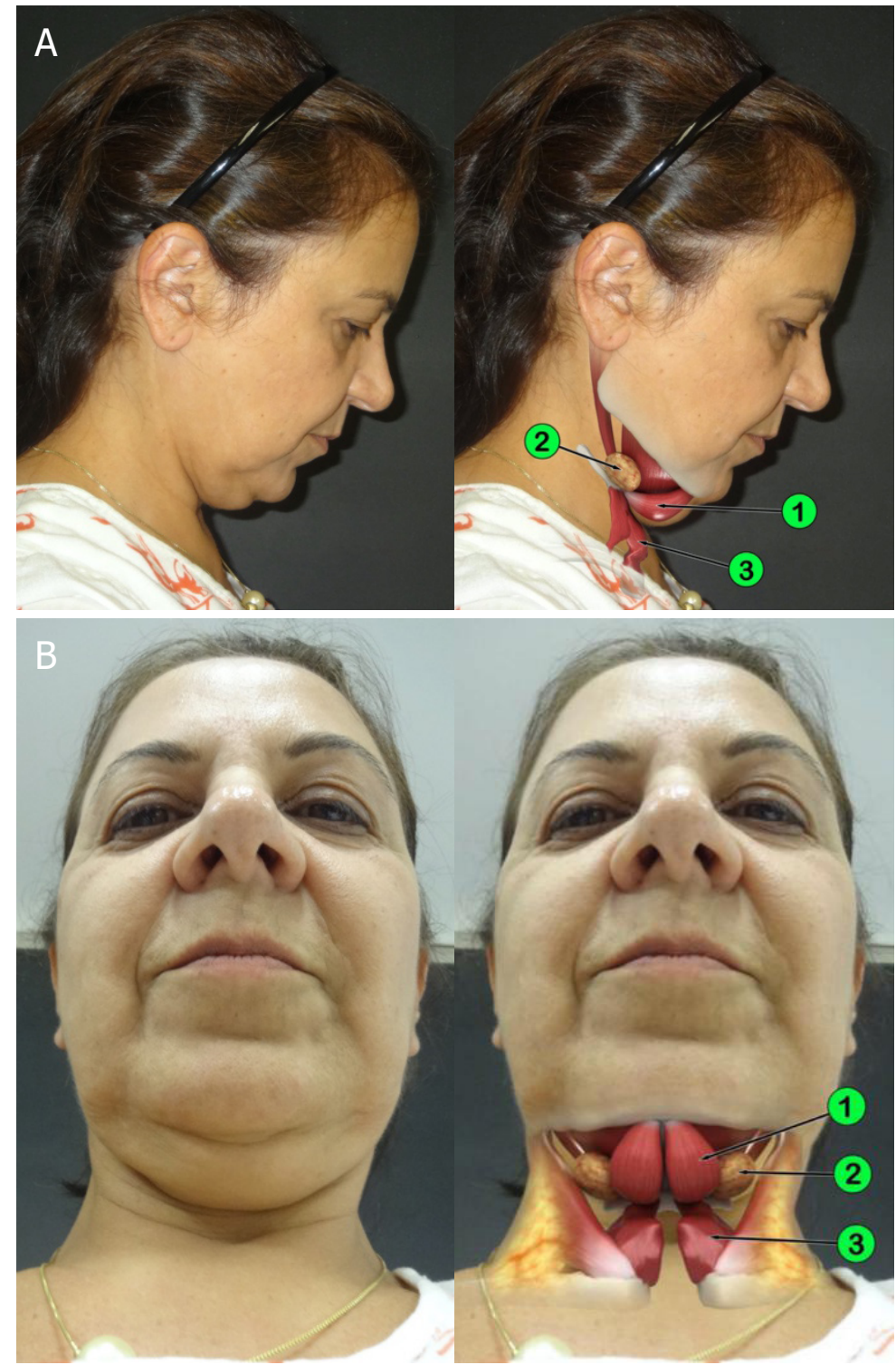

Figure 1. (A) Lateral view of the flexed neck in a 54-year-old woman showing the subplatysmal structures that have an impact on cervical contour: (1) hypertrophied anterior belly of the digastric muscle, (2) enlarged and ptotic submandibular salivary gland, and (3) flaccidity of the sternohyoid muscles; (B) upward view of the flexed neck of the same patient. Fat covering the sternocleidomastoid muscles is also illustrated (yellow)

postoperatively every $8 \mathrm{~h}$ for the first $24 \mathrm{~h}$. In case nausea and vomiting occurs, the causes are thoroughly evaluated and properly treated.

\section{Surgical technique}

A Supplementary Video depicts the main steps of the technique described below.

Neck lift is performed in all patients through a submental incision, which allows for access to the platysma and the supraplatysmal and subplatysmal areas in the suprahyoid and infrahyoid regions. The neck lift is performed alone or in combination with the lifting of the middle third of the face. In some patients, blepharoplasty, gliding brow lift, or endoscopic brow lift is also performed in the same surgical session. 


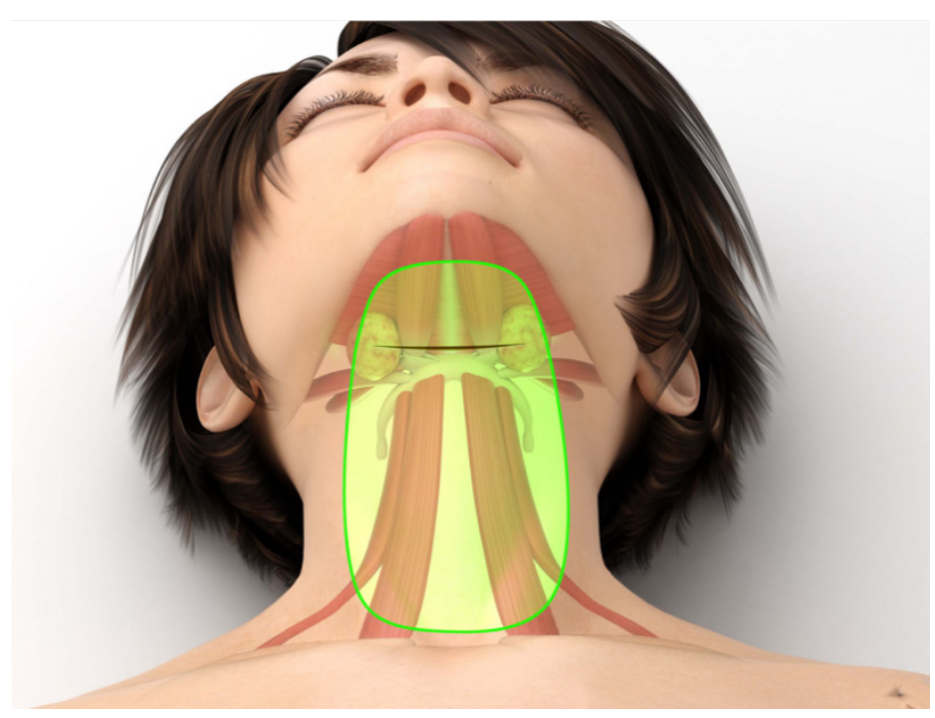

Figure 2. Illustration of the position of the incision for a subplatysmal neck lift (black line, $1.5 \mathrm{~cm}$ anterior to the hyoid) and the initial dissecting area, limited to the central region

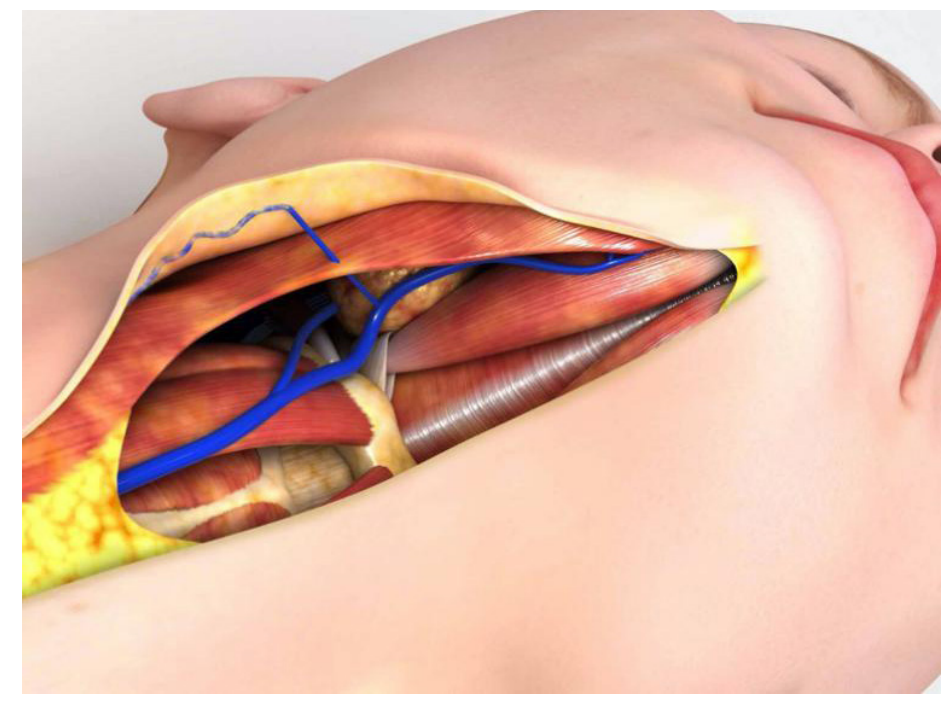

Figure 3. A vein travels from the subcutaneous space towards the submandibular glands passing perpendicularly through the platysma. Blunt dissection facilitates hemostatic control of this vessel

Submental incision is placed about $1.5 \mathrm{~cm}$ anterior to the hyoid bone. This position allows for an easier access to the subplatysmal structures, especially the SMGs, the DMs, and the SHMs. Dissection of the subcutaneous space is initially aimed at creating enough room in the central region of the neck, in a vertical column that spans from about $2 \mathrm{~cm}$ anterior to the submental incision down to about $3 \mathrm{~cm}$ above the sternal notch [Figure 2].

Undermining from the midline to the lateral aspect of the neck should be enough to expose the medial margins of the platysma. A complete skin detachment of the neck at this stage should be avoided since it will certainly lead to more bleeding. In fact, during this dissection, a vein is frequently found on each side of the neck about 4 to $5 \mathrm{~cm}$ from the midline at the level of the SMGs. It originates in the subcutaneous compartment and dives perpendicularly into the platysma towards the SMG. For practical purposes, we call this the perpendicular vein of the platysma [Figure 3]. Severing it leads to inconvenient bleeding. The 
use of a Trepsat blunt dissector, or of a Viterbo's spreader, ensures a safer undermining with less bleeding and less trauma to the flap ${ }^{[14]}$. The use of liposuction or cannulas for blunt dissection should be avoided to prevent trauma of the skin flap and excessive removal of subcutaneous fat.

Next, inventory of the subplatysmal area is initiated. The medial margins of the platysma are exposed by judicial removal of the interplatysmal fat that often covers them, especially in the area corresponding to the anterior bellies of the DMs. During this defatting of the platysma, the surgeon may encounter bleeding from the anterior jugular veins. The platysma is then lifted using the blunt dissectors mentioned above, which allow for a less traumatic access of the subplatysmal compartment. Importantly, this detachment should encompass the areolar tissue close to the superficial layer of the deep cervical fascia (also called the investing deep fascia), so that the muscle flap becomes robust enough to ensure its use in the next steps of the procedure, as described below.

In order to facilitate the visualization of the subplatysmal area, the platysma is retracted using a stitch that secures it attached to the overlying skin ${ }^{[15]}$.

In the infrahyoid region, dissection is then carried out between the superficial fascia, that covers the platysma internally, and the investing deep fascia. With the platysma lifted, the investing deep fascia in the infrahyoid region is usually seen as a light fascia, in contrast with its denser aspect in the perihyoid and suprahyoid segments.

Once the subplatysmal compartment is exposed in its central area, three conditions are analyzed: (1) the volume of its main structures - the DMs, subplatysmal fat, and SMGs; (2) the position of the hyoid bone; and (3) the flaccidity of the SHMs. Plication of the DMs is usually indicated to reestablish the roof of the submental triangle and to reposition a bulging mylohyoid muscle ${ }^{[16,1]}$. It is usually started by applying a stitch with a 3-0 absorbable monofilament to the intermediate tendons of the DMs (Monosyn, $26 \mathrm{~mm}$ cylindric needle, B. Braun, Melsungen AG, Germany; or Caprofyl, $26 \mathrm{~mm}$ cylindric needle, Ethicon, Guaynabo, Puerto Rico), especially when the hyoid needs to be placed to a more superior and posterior position. When the hyoid is found to be well positioned, the plication should start in the anterior bellies of the DMs themselves, a few millimeters anterior to the tendons. When the SHMs are flaccid, the suture should continue inferiorly by encompassing the medial margins of these muscles and reach a level 2 to $3 \mathrm{~cm}$ above the suprasternal notch. This subplatysmal midline muscle plication from the chin to the suprasternal notch redefines the central aspect of the neck [Figure 4].

Given the proximity of the DMs tendons and the SMGs capsules, plication of these muscles normally brings the SMGs closer to the midline. This allows for an easier evaluation of the size and position of the glands and the necessity to partially remove them in order to reduce the subplatysmal volume. Partial resection of each SMG is performed with electrocautery. Once this is done, 5 to 10 units of botulinum toxin type A (Botox, Allergan, Ireland) are injected in the raw glandular surface and a double-layer running absorbable suture (monofilament) encompassing the gland is applied to ensure that neither salivary fistulas nor hematomas will occur (4-0 Monosyn, 26 mm cylindric needle, B. Braun, Melsungen AG, Germany; or Caprofyl, $26 \mathrm{~mm}$ cylindric needle, Ethicon, Guaynabo, Puerto Rico). Finally, the robust platysma flap - lifted as indicate above - is sutured over each SMG with a few absorbable stitches (4-0 Monosyn or Caprofyl) to further increase the sealing of this area. The thicker this flap, the better is its capacity to avoid saliva collections. In selected cases, hypertrophied medial platysmal bands are longitudinally trimmed. Plication of the platysma in a corset manner finalizes this stage of the neck lift with a running absorbable suture [Figure 5]. This suture starts at the level of the hyoid, goes down to about 2 to $3 \mathrm{~cm}$ above the suprasternal notch and returns only to be interrupted about $3 \mathrm{~cm}$ from the mentum to allow for the submental ligament release and detachment of skin over the chin [Figure 6]. During this dissection, 

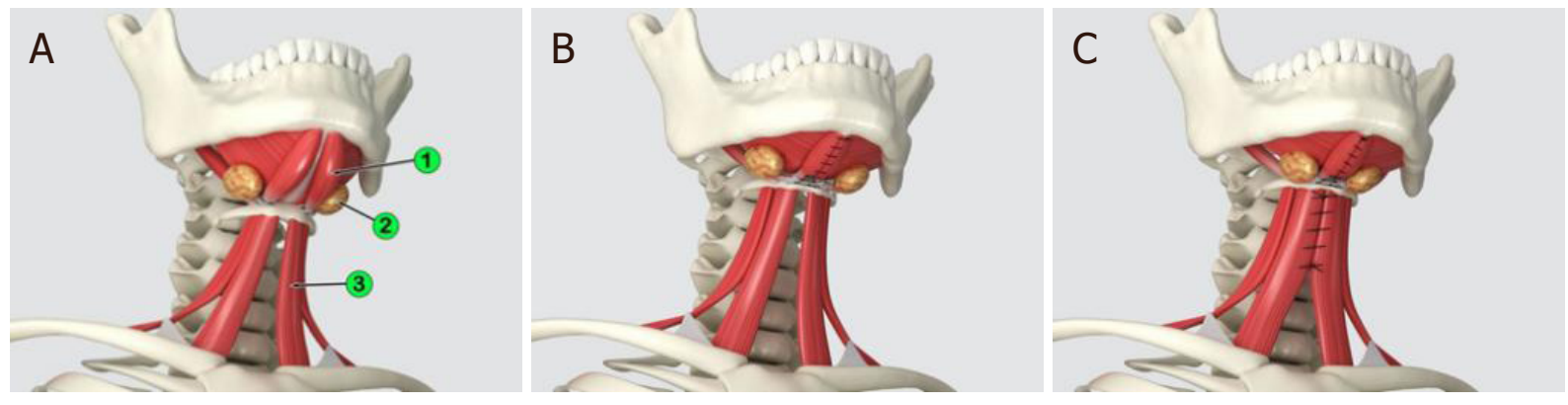

Figure 4. (A) Illustration of subplatysmal findings in the heavy neck: (1) hypertrophied anterior bellies of the digastric muscles, (2) enlarged and ptotic submandibular salivary glands, and (3) flaccid sternohyoid muscles; (B) plication of the digastric muscles; (C) plication of the sternohyoid muscles. A midline subplatysmal corset is obtained, reestablishing a new cervical contour
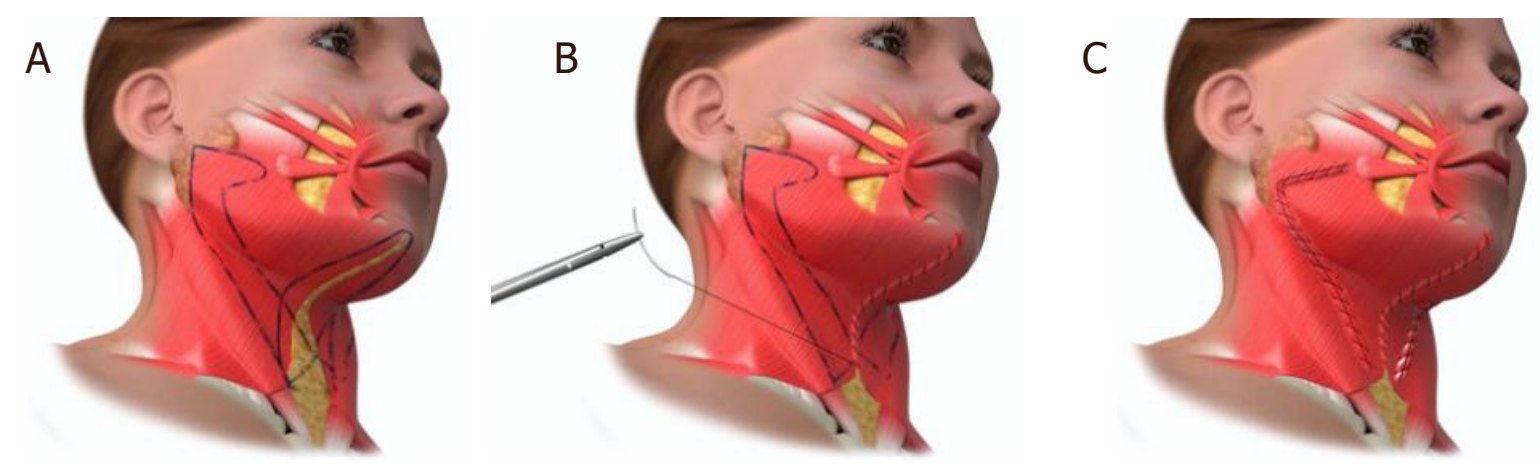

Figure 5. (A) Illustration depicting the platysma plication plan with midline and lateral plications; (B) midline plication refines the subplatysmal structural work; (C) rejuvenating the sternocleidomastoid muscle includes defatting and lateral plication over its anterior margin

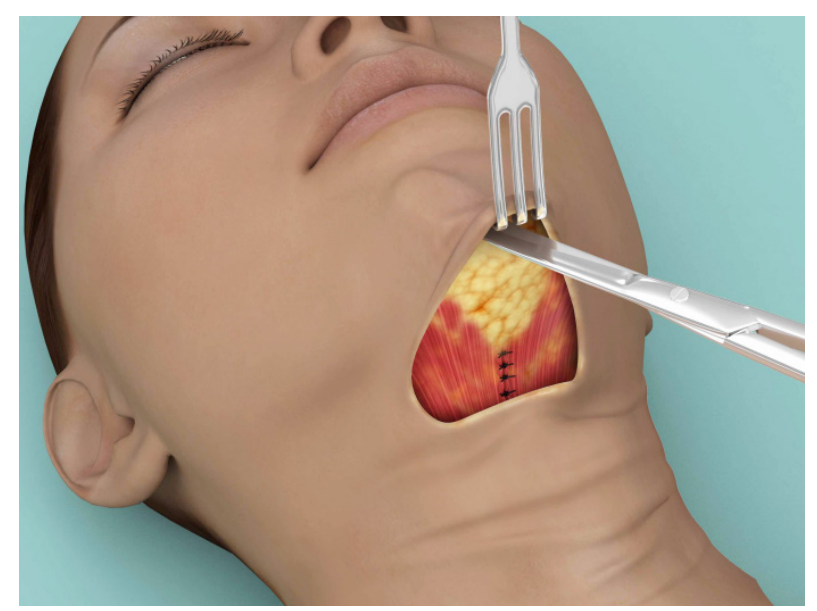

Figure 6. Illustration of retrograde dissection of the chin. Release of the submental ligament allows for adequate leveling of the fat in this area and a complete platysma plication

especial attention should be devoted not to injure the marginal mandibular branch of the facial nerve. Open lipectomy in this area levels the planes allowing for completion of platysma plication.

Lateral access through a periauricular incision permits treatment (rejuvenation) of the SCM. Skin dissection is usually performed after tumescent subcutaneous infiltration and aims at releasing the most inferior cervical creases and at communicating with the anterior compartment created in the previous step 
Table 1. Patient demographics $(N=1,019)$

\begin{tabular}{ll}
\hline No. of women (\%) & $925(90.8)$ \\
No. of men (\%) & $94(9.2)$ \\
Primary neck lift, no. of patients (\%) & $869(85.3)$ \\
Secondary neck lift, no. of patients (\%) & $144(14.1)$ \\
Terciary neck lift, no. of patients (\%) & $6(0.6)$ \\
Combined neck lift and rhytidoplasty, no. of patients (\%) & $939(92.2)$ \\
Neck lift alone, no. of patients (\%) & $80(7.8)$ \\
\hline
\end{tabular}

of the surgery. Once the skin is lifted, fat may be encountered over the SCM. This adipose tissue has two distinct layers: the layer more superficial and easily removed through open liposuction, and the layer closer to the platysma that covers the SCM, with a fibrous aspect, usually treated with Metzenbaum scissors.

If the middle third of the face is to be treated - in the authors' preference usually through a deep plane technique - the SCM is approached after this step, as follows. A stitch with a 3-0 absorbable monofilament (Monosyn, 32 mm, cylindric, 3/8 needle, B. Braun, Melsungen AG, Germany; or Caprofyl, $31 \mathrm{~mm}$ cylindric needle, Ethicon, Guaynabo, Puerto Rico) encompasses the superficial musculoaponeurotic system (SMAS)platysma just anterior to the SCM (2 to $4 \mathrm{~cm}$ bellow the earlobe) and is anchored to the most superior aspect of the platysma-auricular ligament, at the level of the tragus, reestablishing the musculomandibular triangle. A plication is then run just anterior to the SCM border, on a territory anterior to the main branches of the great auricular nerve, aiming to reach the most inferior and medial aspect of the neck [Figure 5]. As the plication is performed, redundant fibrous fatty tissue over the SCM is removed, helping in redefining its anterior border.

After the subplatysmal and platysmal works are concluded, the next step is to adequately distribute the skin over the new structured neck. When needed, excess skin is usually removed through a periauricular incision. In patients with good quality of skin, particularly young ones, this may not be needed. Skin redraping is performed in all patients with the help of the hemostatic net, a surgical tactic previously described ${ }^{[18]}$. The hemostatic net was originally developed to prevent the occurrence of hematomas in rhytidoplasties and cervicoplasties. Its principle is the compulsory closure of all detached areas through the application of several columns of running sutures - with a 5-0 nylon with a $26 \mathrm{~mm}$ semicircular triangular needle - that encompass the skin and the SMAS-platysma (Ethicon, São José dos Campos, Brazil). In addition to closing all dead space and preventing fluid accumulation, the net greatly facilitates skin distribution. The net is usually removed between 48 and $72 \mathrm{~h}$ after surgery.

\section{RESULTS}

Table 1 summarizes the demographics of the 1,019 patients included in this study. Most patients were women $(925,90.8 \%)$. The patients' mean age was 50.6 years (range, 16-81 years). Neck lift was a primary procedure in 869 of patients $(85.3 \%)$.

Most patients underwent a combined rhytidoplasty (939, 92.1\%). Neck lift alone was performed in 80 patients $(7.9 \%)$, of which, 35 (43.7\%) had a periauricular incision to accommodate or excise redundant skin and $45(56.3 \%)$ received an exclusive submental incision.

Nine hundred and thirty-seven patients underwent some type of subplatysmal work (91,9\%) [Table 2]. Partial removal of the SMGs was performed in 782 patients $(76.8 \%)$. Anterior bellies of the DMs were partially resected in 549 patients $(53.9 \%)$ and plicated in 472 patients (46.3\%). SHMs suture was applied in 348 patients (34.1\%) and 784 patients underwent SCMs rejuvenation (76.9\%). All patients received a corset platysmaplasty. 
Table 2. Subplatysmal neck lift procedures among 1,019 patients

\begin{tabular}{lc}
\hline Neck lift procedure & Number of patients (\%) \\
\hline Subplatysmal treatment & $937(91,9 \%)$ \\
Partial resection of the SMGs & $782(76.8 \%)$ \\
Partial resection of DMs & $549(53.9 \%)$ \\
DMs plication & $472(46.3 \%)$ \\
SHMs plication & $348(34.1 \%)$ \\
SCMs rejuvenation & $784(76.9 \%)$ \\
\hline
\end{tabular}

SMG: submandibular glands; DMs: digastric muscles; SHMs: sternohyoid muscles; SCMs: sternocleidomastoid muscles

Figures 7 and 8 illustrate two patients who underwent subplatysmal neck lift.

The most common complication in this series was transient paralysis of the lower lip depressor muscle (79 patients, 7.7\%) [Table 3]. Fluid collection over the parotid gland, possibly indicating sialoma from this gland or a localized seroma, was observed in 11 patients $(1.1 \%)$. Salivary fistula and sialoma from one of the SMGs were diagnosed in $4(0.4 \%)$ and 2 patients $(0.2 \%)$, respectively.

\section{DISCUSSION}

This article summarizes our experience with surgical cervical rejuvenation during the last 10 years. Its main objective is to provide a comprehensive description of the subplatysmal tactics incorporated over time, including two original ones, the SHMs plication and the SCMs rejuvenation. Results were obtained from a quantitative perspective. Subjective data of the many subgroups of patients with these different approaches were not retrieved. Although this may impair the qualitative interpretation of the results, it does not invalidate the main descriptive goal of the present work.

A well-contoured neck is one of the top priorities of patients when seeking facial and cervical rejuvenation. A less than optimal result in the neck usually overshadows an excellent result in the middle third of the face. Recognizing the preoperative anatomical problems of the neck allows for an adequate surgical plan and successful postoperative result.

The neck can be seen as a cylinder that undergoes changes in shape and dimensions with time [Figure 9] ${ }^{[8]}$. It progressively loses height due to the reduction of its intervertebral spaces. A gain in diameter is also observed as a consequence of hypertrophy and malposition of its deep embedded structures, such as subplatysmal fat, DMs, and SMGs. Concomitant cervical spine lordosis pushes these structures anteriorly, augmenting the perception of cervical aging. In parallel, a hyoid bone found in a more anterior and inferior position gives an obtuse and heavy aspect to the neck.

Exploring the subplatysmal region below the hyoid permits the examination of the SHMs and their fascia, often found to be loose. This intraoperative finding explains, in part, the preoperative perception of an increased lower neck diameter. Accumulation of subcutaneous fat and laxity of skin and platysma are frequently responsible for the rest of the enlarged lower neck.

Fat deposition in the subcutaneous space also blurs the definition of the anterior margin of the SCM, often on its entire length, from the musculomandibular triangle to the clavicle.

In recent years we have incorporated the plication of the SHMs as a way to increase the quality of the lower neck contour. The procedure is well indicated particularly in older patients and those who underwent bariatric surgery. This suture is a continuation of the plication of the anterior belly of the DMs, with or without their partial resection, a standard in our practice in the last years. It may start with the 

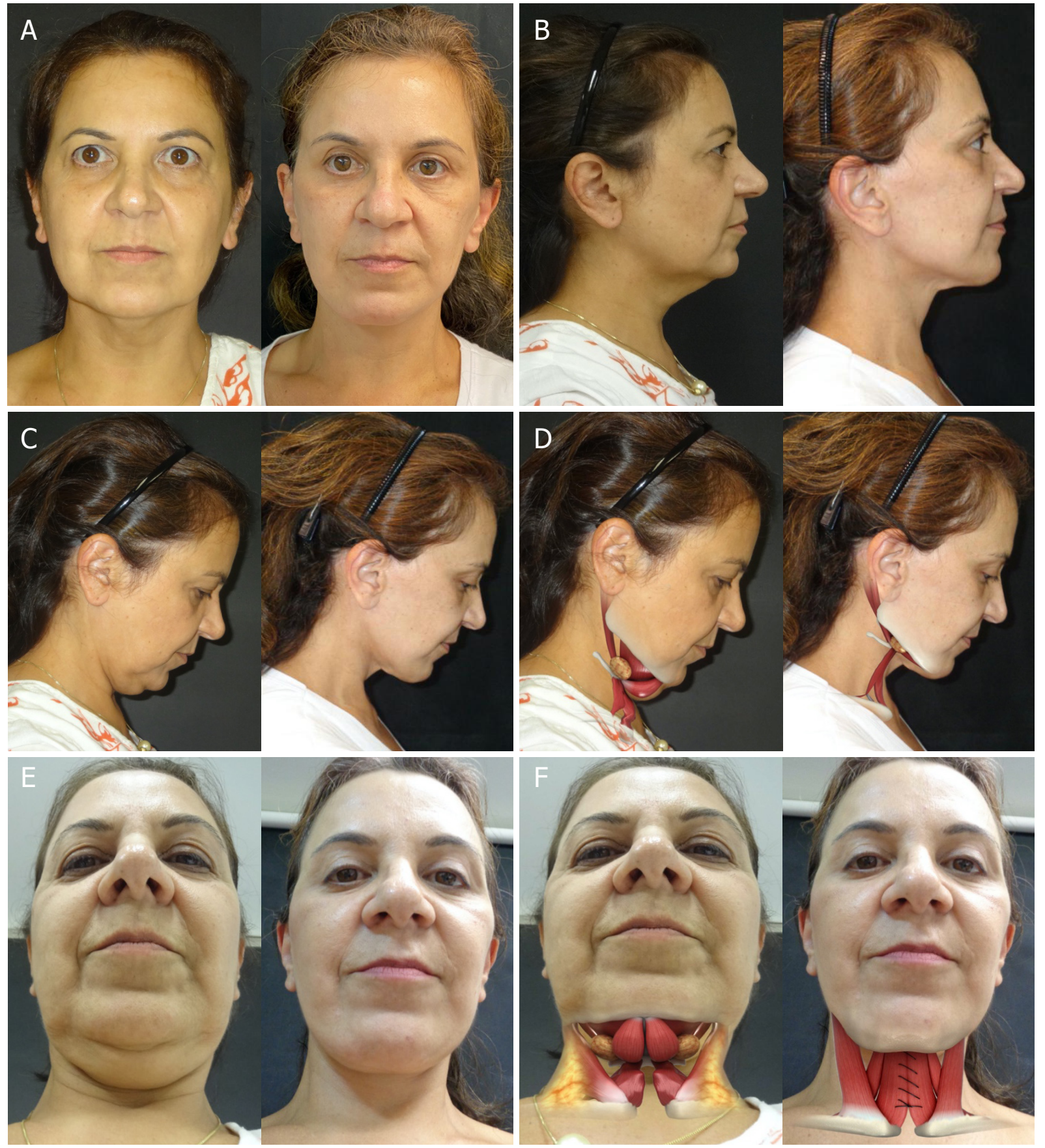

Figure 7. Pre (left) and 2 years postoperatively (right) pictures of a 54-year-old woman who underwent a subplatysmal neck lift. Surgical plan included anterior digastric bellies partial removal and plication, plication of the sternohyoid muscles, partial removal of the submandibular salivary glands, subplatysmal lipectomy, platysma corset, sternocleidomastoid rejuvenation, middle third rhytidectomy with SMAS plication, and hemostatic net. (A) Front view; (B) lateral view with the neck in a neutral position; (C) right lateral view of the flexed neck; (D) right lateral view of the flexed neck with superimposed drawing showing structures treated; (E) upward view of the flexed neck (F) upward view of the flexed neck with superimposed drawing showing structures treated

approximation of the DMs tendons, when there is an indication to a superior and posterior reposition of the hyoid, or in the anterior belly. Since it spans from the chin to about 2 to $3 \mathrm{~cm}$ above the sternal notch, this suture is comparable to the approximation of the rectus abdominis in abdominoplasties, which runs from the xiphoid process to the pubis. It helps to redefine, with a long-lasting capacity, the cervical cylinder in a deep level. This enduring result is credited to the fact that these muscles have well established bone 

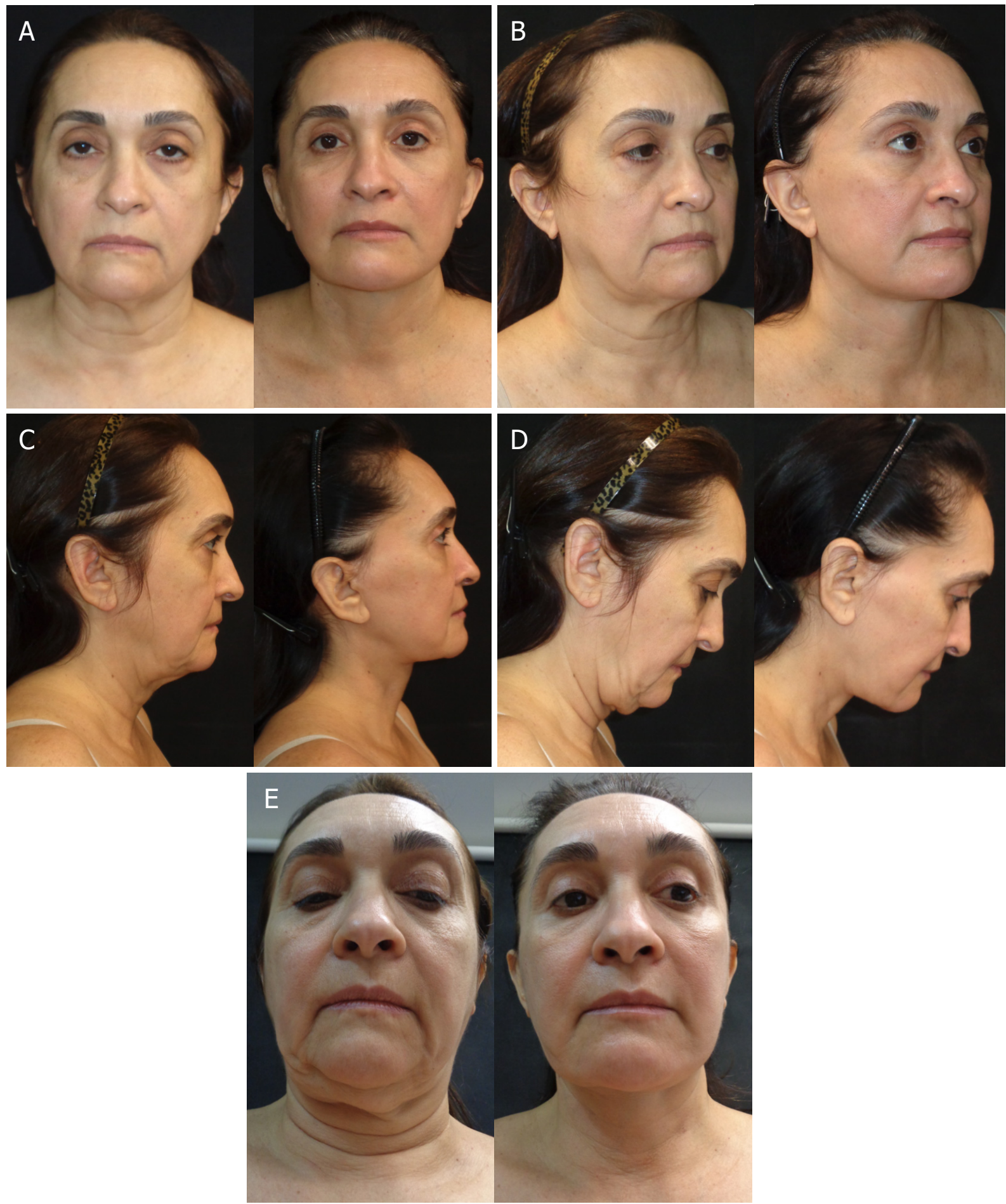

Figure 8. Pre (left) and 1.5 years postoperatively (right) pictures of a 53-year-old woman who underwent a subplatysmal neck lift. Surgical plan included endoscopic brow lift, superior blepharoplasty with treatment of eye lid ptosis, anterior digastric bellies partial removal and plication, plication of the sternohyoid muscles, partial removal of the submandibular salivary glands, subplatysmal lipectomy, platysma corset, sternocleidomastoid rejuvenation, deep plane middle third rhytidectomy, and hemostatic net. (A) Front view; (B) oblique view; (C) lateral view with the neck in a neutral position; (D) right lateral view of the flexed neck; (E) upward view of the flexed neck

insertions. The corset of the platysma, as described by Feldman ${ }^{[6]}$, finishes the work in the anterior neck. Importantly, there is no need for excessive tensioning in this suture, since the deep subplatysmal structures were already trimmed and repositioned. 
Table 3. Complications in neck lifts among 1,019 patients

\begin{tabular}{lc}
\hline Type of complication & Number of patients (\%) \\
\hline Weakness of the lower lip depressor & $79(7.7)$ \\
Submental scar revision & $14(1.3)$ \\
Parotid gland sialoma & $11(1.0)$ \\
Hematoma (> 20 mL blood collection) & \\
During first 48 h (hemostatic net) & $0(0)$ \\
After 48 h (i.e., after net removal) & $7(0.6)$ \\
Salivary fistula from SMGs & $4(0.4)$ \\
Sialoma of SMGs & $2(0.2)$ \\
\hline
\end{tabular}

*There were no instances of subplatysmal hematomas. SMGs: submandibular salivary glands
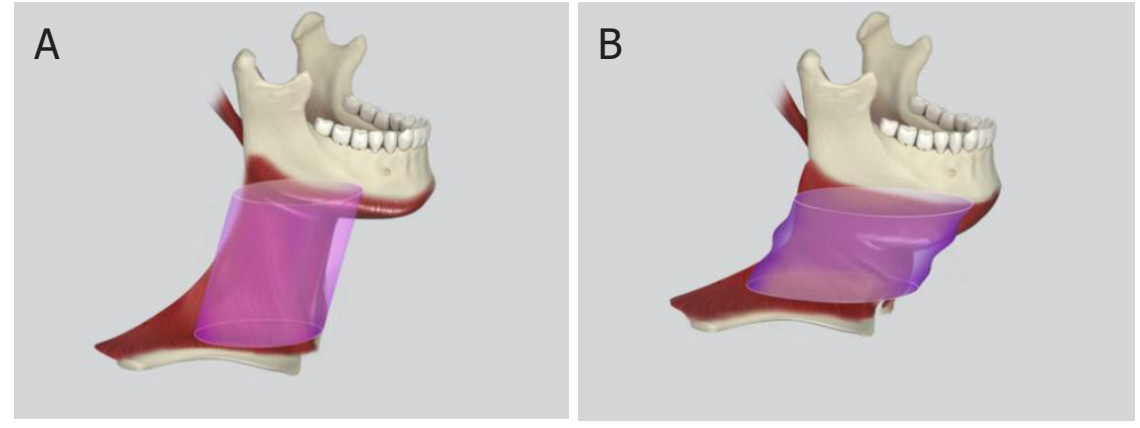

Figure 9. The neck is comparable to a cylinder that loses height and increases diameter with time. Cervical spine lordosis, increased volume, and platysma and skin laxity are on the basis of the aging process. (A) Illustration of a young neck with a superimposed cylinder higher and with a smaller diameter then the aged one shown on illustration (B)

The SCMs account for most of the lateral aspect of the neck. Their importance in defining the youthful neck has been described by Ellenbogen over 40 years ago ${ }^{[5]}$. However, little attention has been dedicated to specific tactics to reestablish the visibility of their anterior margins. In fact, many approaches are competent in treating thin patients, in whom the SCMs are already well defined, but bring incomplete solutions for those with a heavy neck. For instance, treating the cervical region exclusively through a lateral access (i.e., without a submental incision) inevitably limits the access to the lower aspect of the SCMs. Techniques that include the platysma flap posterior traction (anchoring it to the mastoid fascia) superimpose the platysma over the SCM, at least in its superior half, adding volume where there should be less. SCM rejuvenation, as described in this study, ensures an adequate cleaning of the anterior margin through its defatting and a lateral-to-middle platysma plication. This tensioning of the platysma over the SCM complements the anterior corset. In fact, when the platysma is only tensioned through the midline, the SCM ends up with a less then optimal definition. Furthermore, the initial stitch placed in the platysma-auricular ligament helps to reestablish the musculomandibular triangle, an important hallmark of the youthful neck.

Skin distribution has always been a challenge in neck and face lifts. The use of the hemostatic net has greatly contributed to solving this issue, besides preventing the formation of hematomas. In our practice, the net is used in all cervicoplasty and rhytidoplasty patients with no hematomas observed in the first $48 \mathrm{~h}$, even in situations complicated by postoperative hypertension, vomiting, or psychomotor agitation. Early mobilization and discharge are other advantages of this tactic.

Complications in this series were similar in incidence and severity to those of other authors ${ }^{[19,20]}$. Transient lower lip depressor weakness was the most common adverse event. Direct dissection over the territory of lower lip muscles, maneuvers on the path of the marginal mandibular and cervical branches of the facial nerve, and manipulation of the platysma are possible causes to this observation. 
Unaesthetic submental scar was the second most common complaint, leading to surgical revision. The unpleasant aspect of this scar was related more to its hypertrophy aspect than to its position. In fact, placing the incision over the submental ligament - as recommended by many authors and as we used to do in our early cases - may lead to the intensification of the submental crease, besides limiting the access to the inferior neck and to subplatysmal structures. Furthermore, optimal exposure of the surgical field is critical for adequate control of bleeding, especially when resecting the SMGs.

Fluid accumulation on the territory of the parotid gland, possibly corresponding to a sialoma, was observed in patients who underwent SMAS plication and was possibly the result of the several stitches placed over the parotid fascia. Treatment included drainage, injection of botulinum toxin type A, local quilting suture to close the dead space (as performed in the hemostatic net), and compressive dressings. Since we adopted the deep plane approach, we have not experienced this complication.

Salivary fistulas and sialomas from the SMGs are possibly the most strenuous complications to treat. As described above in "Surgical Technique", prevention with surgical maneuvers and injection of botulinum toxin type A are paramount. Of note, care should be taken not to inject the toxin into the swallowing muscles around the SMGs, since this may lead to prolonged and severe dysphagia, as we have observed in one patient. In fact, after this case, we stopped using botulinum toxin type A for almost a year. During this time, we observed a surge in cases of salivary fistulas.

Treatment of a salivary fistula is usually time consuming and includes placing a drain, compressive dressings, and dietary restrictions. Pharmacological measures to reduce saliva production may also help in the treatment. Sublingual use of $1 \%$ atropine sulfate ${ }^{[21]}, 1.5 \mathrm{mg}$ scopolamine dermal patches, and oral intake of trihexyphenidyl $2 \mathrm{mg}^{[22]}$ are among the options. Botulinum toxin injection under ultrasound guidance, albeit possible, is an alternative that always carries risks of arterial bleeding from the gland and is not the preferred way of treating this complication.

Subcutaneous hematoma is no longer the most common complication in this type of surgery due to the introduction of the hemostatic net. No bleeding was observed during the time the net was kept in place. A few cases occurred after it was removed and were correlated with restlessness, hypertension, and nausea and vomiting. Treatment was aspiration and application of another hemostatic net in the hematoma area. Although there is a report in the literature of a postoperative subplatysmal arterial bleeding from the $\mathrm{SMG}^{[23]}$, no such event occurred in the present series. This is certainly in part due to the hemostatic suture over the SMGs.

Limited transient difficulties to swallow may be observed in the first few days after a subplatysmal neck lift. Warning patients about these symptoms may ease the understanding of the normal postoperative recovery.

Finally, although many perceive the subplatysmal neck lift as a surgical procedure that would often lead to overcorrection $^{[24,25]}$, this is not what our experience has shown. As in other plastic surgeries, care must be taken to adequately find the balance between the removal of tissue and the volume kept. Maintaining the subcutaneous fat and a conservative resection of the fibrous fatty tissue over the hyoid are among some of the measures that ensure equilibrium and grace to the final result.

In conclusion, subplatysmal approach for neck lift can be safely applied to reestablish the cervical contour. Rejuvenating the SCMs and treating the inferior neck with plication of the SHMs are new tactics to optimize results. Additional qualitative analysis may be useful in measuring the benefits of these techniques. Attention to surgical steps and maneuvers is key to ensure successful outcomes. 


\section{DECLARATIONS}

\section{Authors' contributions}

Contributed to the article: Auersvald LA, Auersvald A

\section{Availability of data and materials}

Not applicable.

\section{Financial support and sponsorship}

None.

\section{Conflict of interest}

All authors declared that there are no conflicts of interest.

\section{Ethical approval and consent to participate}

All methodologies and study protocols were approved by the Ethics Committee (Hospital Lipoplastic).

\section{Consent for publication}

Written consent for publication was obtained for all images.

\section{Copyright}

(C) The Author(s) 2021.

\section{REFERENCES}

1. Connell BF. Neck contour deformities: the art, engineering, anatomic diagnosis, architectural planning, and aesthetics of surgical correction. Clin Plast Surg 1987;14:683-92.

2. Mejia JD, Nahai FR, Nahai F, Momoh AO. Isolated management of the aging neck. Semin Plast Surg 2009;23:264-73

3. Rohrich RJ, Rios JL, Smith PD, Gutowski KA. Neck rejuvenation revisited. Plast Reconstr Surg 2006;118:1251-63.

4. Auersvald A, Auersvald LA, Uebel CO. Subplatysmal neck lift: a retrospective analysis of 504 patients. Aesth Surg J 2016;37:1-11.

5. Ellenbogen R, Karlin JV. Visual criteria for success in restoring the youthful neck. Plast Reconstr Surg 1980;66:827-37.

6. Feldman JF. Neck lift. 1st ed. St. Louis, MO: Quality Medical Publishing, Inc; 2006.

7. Auersvald A, Auersvald LA. Management of the submandibular gland in neck lifts: indications, techniques, pearls, and pitfalls. Clin Plast Surg 2018;45:507-25.

8. Ramirez OM. Multidimensional evaluation and surgical approaches to neck rejuvenation. Clin Plast Surg 2014;41:99-107.

9. Narasimhan K, Stuzin JM, Rohrich RJ. Five-step neck lift: integrating anatomy with clinical practice to optimize results. Plast Reconstr Surg 2013;132:339-50.

10. Marten T, Elyassnia D. Neck lift: defining anatomic problems and choosing appropriate treatment strategies. Clin Plast Surg 2018;45:455-84

11. Marten T, Elyassnia D. Short scar neck lift: neck lift using a submental incision only. Clin Plast Surg 2018;45:585-600.

12. Jacono A, Bryant LM. Extended deep plane facelift: incorporating facial retaining ligament release and composite flap shifts to maximize midface, jawline and neck rejuvenation. Clin Plast Surg 2018;45:527-54.

13. Bravo FG. Reduction neck lift: the importance of the deep structures of the neck to the successful neck lift. Clin Plast Surg 2018;45:485-506

14. Viterbo F. Improved blunt dissectors for greater safety in face lift surgery. Plast Reconstr Surg 2013;131:858e-9e.

15. Auersvald A, Auersvald LA. Innovative tactic in submandibular salivary gland partial resection. Plast Reconstr Surg Glob Open 2014;2:e274.

16. Ramirez OM, Robertson KM. Comprehensive approach to rejuvenation of the neck. Facial Plast Surg 2001;17:129-40.

17. Labbé D, Giot JP, Kaluzinski E. Submental area rejuvenation by digastric corset: anatomical study and clinical application in 20 cases. Aesthetic Plast Surg 2013;37:222-31.

18. Auersvald A, Auersvald LA. Hemostatic net in rhytidoplasty: an efficient and safe method for preventing hematoma in 405 consecutive patients. Aesthetic Plast Surg 2014;38:1-9.

19. Feldman JJ. Neck lift my way: an update. Plast Reconstr Surg 2014;134:1173-83.

20. Benslimane F, Kleidona IA, Cintra HPL, Ghanem AM. Partial removal of the submaxillary gland for aesthetic indications: a systematic review and critical analysis of the evidence. Aesthetic Plast Surg 2020;44:339-48.

21. Dias BLS, Fernandes AR, Maia HS Filho. Treatment of drooling with sublingual atropine sulfate in children and adolescents with cerebral 
palsy. Arq Neuropsiquiatr 2017;75:282-7.

22. Reid SM, Westbury C, Guzys AT, Reddihough DS. Anticholinergic medications for reducing drooling in children with developmental disability. Dev Med Child Neurol 2020;62:346-53.

23. Mendelson BC, Tutino R. Submandibular gland reduction in aesthetic surgery of the Neck: review of 112 consecutive cases. Plast Reconstr Surg 2015;136:463-71.

24. Baker, DC. Face lift with submandibular gland and digastric muscle resection: radical neck rhytidectomy, Aesth Surg J 2006;1:85-92.

25. Ceravolo MP. Invited discussion on: aesthetic submandibular gland partial resection-a systematic review and critical analysis of the evidence. Aesthetic Plast Surg 2020;44:349-53. 